\title{
Degree Sums for Edges and Cycle Lengths in Graphs
}

\author{
Stephan Brandt ${ }^{1, *}$ and Henk Jan Veldman ${ }^{2}$ \\ ${ }^{1}$ FREIE UNIVERSITÄT BERLIN \\ FB MATHEMATIK \& INFORMATIK, WE 2 \\ ARNIMALLEE 3 \\ 14195 BERLIN, GERMANY \\ E-mail: brandt@math.fu-berlin.de \\ ${ }^{2}$ FACULTY OF APPLIED MATHEMATICS \\ UNIVERSITY OF TWENTE \\ P.O. BOX 217 \\ 7500 AE ENSCHEDE, THE NETHERLANDS \\ E-mail: h.j.veldman@math.utwente.nl
}

Received October 30, 1995

\begin{abstract}
Let $G$ be a graph of order $n$ satisfying $d(u)+d(v) \geq n$ for every edge $u v$ of $G$. We show that the circumference - the length of a longest cycle - of $G$ can be expressed in terms of a certain graph parameter, and can be computed in polynomial time. Moreover, we show that $G$ contains cycles of every length between 3 and the circumference, unless $G$ is complete bipartite. If $G$ is 1-tough then it is pancyclic or $G=K_{r, r}$ with $r=n / 2$. ( 1997 John Wiley \& Sons, Inc. J Graph Theory 25: 253-256, 1997
\end{abstract}

Keywords: degree sum, circumference, cycle hamiltonian graph, pancyclic graph, tough graph, closure

We use [3] for terminology and notation not defined here, and consider finite, simple, undirected graphs only.

An early sufficient degree sum condition on a graph to be hamiltonian is the following due to Ore.

\footnotetext{
* Partially supported by Deutsche Forschungsgemeinschaft (DFG), Grant We 1265. Most of this research was performed while the first author was at the University of Twente.
}

(c) 1997 John Wiley \& Sons, Inc.

CCC 0364-9024/97/040253-04 
Theorem 1 (Ore [7]). Every graph $G$ of order $n \geq 3$ satisfying $d(u)+d(v) \geq n$ for every pair of non-adjacent vertices $u$, $v$ is hamiltonian.

We investigate the cycle lengths occurring in a graph where every pair of adjacent vertices $u, v$ satisfies $d(u)+d(v) \geq n$. Certainly, we cannot hope any more that every such graph is hamiltonian, as the condition applies to every complete bipartite graph - hence also to the star $K_{1, n-1}$, which contains no cycle at all. But we will show that we can express the exact value of the circumference - the length of a longest cycle - of such a graph in terms of a certain parameter of the graph. Moreover, we can determine its circumference in polynomial time (while this problem is NP-hard within the class of graphs with minimum degree exceeding $n / 2-\sqrt{n}$, see [5]), and the graph contains cycles of every length between 3 and the circumference, unless it is complete bipartite.

Define the independent deficiency of a non-complete graph $G$ as

$$
\phi(G)=\max _{S}|S|-|N(S)|+1,
$$

where the maximum ranges over all non-empty sets $S$ of independent vertices of $G$ with $S \cup$ $N(S) \neq V(G)$. Set

$$
s(G)=\max \{0, \phi(G)\} .
$$

The following simple observation gives an upper bound for the length of a longest cycle.

Lemma 1. The circumference of any graph $G$ of order $n$ without isolated vertices is at most $n-s(G)$.

In this note we will show that if the degree sum $d(u)+d(v) \geq n$ for every edge $u v$ in $G$, then the circumference is precisely $n-s(G)$, and $G$ contains cycles of all lengths $l$ for $3 \leq l \leq n-s(G)$, unless $G$ is complete bipartite. There are several necessary conditions known for a graph to be hamiltonian. The graph $G$ must satisfy $\phi(G) \leq 0, G$ must be 1-tough (i.e., the number of components of $G-S$ is at most $|S|$ for every vertex cut $S$ ), and $G$ must be path-tough (i.e., every vertex-deleted subgraph has a spanning path). Note that the conditions in the sequence are increasing in strength.

Theorem 2. Let $G \neq K_{1, n-1}$ be a graph of order $n$ without isolated vertices. If the degree sum $d(u)+d(v) \geq n$ for every edge $u v \in E(G)$ then the circumference of $G$ equals $n-s(G)$.

In general, the circumference of a graph $G$ of order $n$ need not be equal to $n-s(G)$, as shown by any 1 -tough nonhamiltonian graph.

Theorem 3. Let $G$ be a graph of order $n$. If every edge uv satisfies $d(u)+d(v) \geq n$ then $G$ contains cycles of every length between 3 and the circumference of $G$, unless $G$ is a complete bipartite graph.

A graph is called pancyclic if it contains cycles of all lengths $l$ for $3 \leq l \leq n$. Since every 1-tough graph satisfies $\phi \leq 0$ we obtain the following consequence.

Corollary 1. Every 1-tough graph $G$ of order $n \geq 2$ satisfying $d(u)+d(v) \geq n$ for every edge uv is pancyclic or $G=K_{n / 2, n / 2}$.

As Theorem 1, Theorem 2 generalizes the well-known theorem of Dirac [4] that every graph $G$ of order $n \geq 3$ with $\delta(G) \geq n / 2$ is hamiltonian, since it is easily seen that such a graph has $\phi(G) \leq 0$. Corollary 1 resembles a theorem of Jung [6] stating that every 1-tough graph $G$ of order $n \geq 11$ satisfying $d(u)+d(v) \geq n-4$ for every pair of non-adjacent vertices $u, v$ is 
hamiltonian. For odd $n \geq 11$ the join of $K_{(n-5) / 2}$ and $\bar{K}_{(n-7) / 2} \cup H$, where $H$ is the unique graph with degree sequence $(1,1,1,3,3,3)$, shows that the inequality in Jung's result cannot be relaxed [6]. The same graphs show that the inequality $d(u)+d(v) \geq n$ in Corollary 1 and Theorem 2 is sharp.

For proving Theorem 2 we need tools from hamiltonian graph theory. A powerful idea is the concept of the closure of a graph due to Bondy and Chvátal [2]. Define the $i$-closure $\operatorname{cl}_{i}(G)$ as the graph obtained from $G$ by iteratively joining independent vertices $x, y$ of degree sum $d(x)+d(y) \geq i$ by an edge until in the resulting graph every pair of independent vertices has degree sum less than $i$. Bondy and Chvátal observed that the $i$-closure is unique, and that a graph is hamiltonian if and only if its $n$-closure is hamiltonian. An easy generalization is that the circumference of $G$ equals the circumference of $\operatorname{cl}_{n}(G)$ which we will show next using standard techniques.

Theorem 4. If $G$ is a graph of order $n$ then the circumference of $G$ equals the circumference of $\operatorname{cl}_{n}(G)$.

Proof. It suffices to prove that adding an edge between two non-adjacent vertices $u$ and $v$ with $d(u)+d(v) \geq n$ cannot increase the circumference of the graph.

Assume, otherwise, that the circumference of $G+u v$ exceeds the circumference of $G$. Certainly, every longest cycle $C$ of $G+u v$ must contain the edge $u v$. Hence $G$ contains a path $P$ from $u$ to $v$ which contains all edges of $C$ except $u v$. Define sets $A=\left\{x: x \in V(P), u x^{+} \in\right.$ $E(G)\} \cup\{x: x \in V(G)-V(P), u x \in E(G)\}$, where $x^{+}$is the successor of $x$ on $P$, and $B=\{x: x \in V(G), v x \in E(G)\}$. As $|A|+|B| \geq n$ and $|A \cup B| \leq n-1$ since $v \notin A \cup B$, we get that $A \cap B \neq \emptyset$, implying that there is a cycle in $G$ which is at least as long as $C$.

Note that the $n$-closure of a graph with degree sum $d(u)+d(v) \geq n$ for every edge $u v$ is a graph with the same degree sum property, since the degree sums of the inserted edges exceed $n$ and the degree sums of edges do not decrease in the closure process. In the proof of Theorem 2 we use a sufficient condition due to Pósa for a graph to be hamiltonian.

Theorem 5 (Pósa [8]). Let $d_{1} \leq d_{2} \leq \cdots \leq d_{n}$ be the degree sequence of a graph $G$ of order $n \geq 3$. If $d_{i} \geq i+1$ for every $i<n / 2$ then $G$ is hamiltonian.

We are now prepared to prove our main result.

Proof of Theorem 2. Consider the $n$-closure $G^{\prime}$ of $G$. We claim that for every pair of vertices $u, v$ of $G^{\prime}$ with $d(u) \leq d(v)$ we have $N(u) \cup\{u, v\} \subseteq N(v) \cup\{u, v\}$. Indeed, assume that $u$ has a neighbor $x \neq v$ which is not a neighbor of $v$. As $d(u) \leq d(v)$ and $d(u)+d(x) \geq n$ we get $d(v)+d(x) \geq n$, so $v x \in E\left(G^{\prime}\right)$, contradicting our assumption. Delete $s(G)\left(=s\left(G^{\prime}\right)\right)$ vertices with the smallest degrees in $G^{\prime}$ to obtain a graph $G^{\prime \prime}$. We will show that $G^{\prime \prime}$ satisfies the condition of Pósa (Theorem 5) and is therefore hamiltonian. By Lemma 1 and Theorem 4 this completes the proof.

Indeed, let $d_{1} \leq d_{2} \leq \cdots \leq d_{n-s(G)}$ be the degree sequence of $G^{\prime \prime}$. If $d_{i} \leq i$ for an index $i<(n-s(G)) / 2$ then for the set $S$ of the $i+s(G)$ vertices of smallest degree in $G^{\prime}$, which is clearly independent, we have $|N(S)| \leq i$ by the initial observation, and therefore $|S|-|N(S)|+1 \geq s(G)+1$ and $|S|+|N(S)|<n$, a contradiction to the definition of $\phi(G)$.

To compute the circumference of a graph $G$ satisfying the hypothesis of Theorem 2, we can proceed along the lines of the proof. First compute the $n$-closure $G^{\prime}$ of $G$ (this can be done in time $\mathcal{O}\left(n^{4}\right)$ ) and sort the vertices by degree. Determine $\phi\left(G^{\prime}\right)$ by taking the maximum of $\left|S_{i}\right|-\left|N\left(S_{i}\right)\right|+1$, where $S_{i}$ denotes the set of the $i$ smallest degree vertices of $G^{\prime}$, and the 
maximum ranges over all $S_{i}$ which are independent and satisfy $S_{i} \cup N\left(S_{i}\right) \neq V\left(G^{\prime}\right)$. With a slightly more careful reasoning we can actually find a longest cycle of $G$ in polynomial time.

Zhang [9] defined the critical independence number of a graph $G$ as

$$
\alpha_{c}(G)=\max _{S}|S|-|N(S)|,
$$

where the maximum ranges over all independent sets $S$ (including $\emptyset$, so that $\alpha_{c}(G) \geq 0$ ). He proved that $\alpha_{c}(G)$ can be computed in polynomial time for any graph $G$. As $\alpha_{c}(G)$ is very much like $s(G)$, in fact $\alpha_{c}(G) \leq s(G) \leq \alpha_{c}(G)+1$ holds, it seems likely that $s(G)$ is computable for any graph $G$ in polynomial time. This would be another approach to computing the circumference of graphs satisfying our hypothesis.

To verify that the graphs satisfying the hypothesis of Theorem 2 contain cycles of every length between 3 and $n-s(G)$ we need the following famous result due to Bondy.

Theorem 6 (Bondy [1]). If $G$ is a hamiltonian graph of order $n$ with at least $n^{2} / 4$ edges then $G$ is pancyclic or $G=K_{n / 2, n / 2}$.

Proof of Theorem 3. Let $C$ be a longest cycle of $G$ having length $k$, and consider the edges of $C$. The endvertices of no such edge have a common neighbor in $V(G)-V(C)$, so the degree sum $d_{v C}(u)+d_{v C}(v) \geq k$ for every edge $u v$ of $C$. This implies that the subgraph $H$ of $G$ induced by $V(C)$ has at least $k^{2} / 4$ edges. By Theorem 6 the proof is complete unless $H=K_{k / 2, k / 2}$.

Now assume that $H$ is complete balanced bipartite and let $A \cup B$ be the bipartition of $H$. Observe that no vertex in $V(G)-V(H)$ is adjacent to both ends of an edge in $H$. So, by the degree sum requirement applied to the edges in $H$, every vertex in $V(G)-V(H)$ must be adjacent to precisely one end of every edge in $H$, i.e., every vertex in $V(G)-V(H)$ is either adjacent to every vertex of $A$ or to every vertex of $B$. If there are two vertices $x, y$ in $V(G)-V(H)$ such that $x$ is adjacent to the vertices in $A$ and $y$ to the vertices in $B$, then $G$ has a $(k+2)$-cycle through $x, y$ and the vertices in $H$, contradicting circumference $k$. So all vertices in $V(G)-V(H)$ are adjacent to all vertices on one side of the bipartition of $H$, say to $A$. If $G-V(H)$ contains an edge then we obtain a $(k+1)$-cycle through this edge and $k-1$ vertices of $H$, which is again a contradiction. So, indeed, $G$ is complete bipartite.

\section{References}

[1] J. A. Bondy, Pancyclic graphs I. J. Combinatorial Theory B 11 (1971), 80-84.

[2] J. A. Bondy and V. Chvátal, A method in graph theory. Discrete Math. 15 (1976), 111-135.

[3] J. A. Bondy and U. S. R. Murty, Graph theory with applications, Macmillan, London and Elsevier, New York (1976).

[4] G. A. Dirac, Some theorems on abstract graphs. Proc. London Math. Soc. (3) 2 (1952), 69-81.

[5] R. Häggkvist, On the structure of non-hamiltonian graphs I. Comb., Prob. and Comp. 1 (1992), 27-34.

[6] H. A. Jung, On maximal circuits in finite graphs. Ann. Discrete Math. 3 (1978), 129-144.

[7] O. Ore, Note on Hamiltonian circuits. Amer. Math. Monthly 67 (1960), 55.

[8] L. Pósa, A theorem concerning Hamilton lines. Magyar Tud. Akad. Mat. Kutató Int. Közl. 7 (1962), 225-226.

[9] C.-Q. Zhang, Finding critical independent sets and critical vertex subsets are polynomial problems. SIAM J. Disc. Math. 3 (1990), 431-438. 